\title{
Microdrilling Demonstrates Superior Patient-Reported Outcomes and Lower Revision Rates Than Traditional Microfracture: A Matched Cohort Analysis
}

\author{
Alexander Beletsky, M.D., Neal B. Naveen, B.S., Tracy Tauro, B.A., \\ Taylor M. Southworth, B.S., Jorge Chahla, M.D., Ph.D., Nikhil N. Verma, M.D., \\ Adam B. Yanke, M.D., and Brian J. Cole, M.D.
}

\begin{abstract}
Purpose: The purpose of this study was to compare patient-reported outcomes and revision rates between the standard microfracture awl versus the microdrilling technique. Methods: Microfracture patients were queried from a singleinstitution database between 2001 and 2016. Patient-reported outcome measure data were collected at preoperative and 6- and 12-month time points, inclusive of the International Knee Documentation Committee (IKDC) score, Short Form 12 (SF12) Physical Component Score (PCS) and Mental Component Score, and all Knee Injury and Osteoarthritis Outcome Score (KOOS) subscales. A matching algorithm based on previous procedures, lesion size, and demographic factors created 2 technique-based cohorts. Outcomes including revision rates and both statistically and clinically significant differences (i.e., the minimally clinically important difference [MCID]) between awl and microdrill cohorts were compared using univariate statistics. Results: A total of 68 patients (aged $32.0 \pm 13.1$ years, 48.5\% female, body mass index $26.7 \pm 5.3 \mathrm{~kg} / \mathrm{m}^{2}$ ), with 34 patients in each group, were included after the match. At 6 months, the microdrilling group demonstrated significantly greater levels of improvement than the awl group on the IKDC, SF12 PCS, and KOOS Pain, Symptom, Sport, and Quality of Life $(P<.04)$, although differences at 1 year were only maintained on the SF12 PCS instrument $(P<.001)$. With respect to MCID achievement, the microdrilling group demonstrated greater achievement rates at 6 months on the IKDC, KOOS Pain, and KOOS Sport $(P<.04)$. The awl group demonstrated a higher rate of revision surgery $(P=.02)$ within 3 years of follow-up and a greater likelihood to require multiple subsequent procedures (41.1\% vs $17.6 \%, P=.03)$. Conclusions: Microdrilling demonstrated superior outcomes relative to traditional microfracture awl techniques with respect to patient-reported outcomes at 6 months and revision rates within 3 years of followup. In addition, clinically meaningful differences were evident at 6 months in the microdrilling group. Level of Evidence: Level III, retrospective comparative study.
\end{abstract}

$\mathbf{F}$ ull-thickness lesions of the articular cartilage present unique challenges for the orthopedic surgeon to address. As a material, articular cartilage is relatively hypodense with limited vascular supply,

From the Division of Sports Medicine, Midwest Orthopaedics at Rush, Rush University Medical Center, Chicago, Illinois, U.S.A.

The authors report that they have no conflicts of interest in the authorship and publication of this article. Full ICMJE author disclosure forms are available for this article online, as supplementary material.

Received April 18, 2020; accepted October 21, 2020.

Address correspondence to Nikhil N. Verma, M.D., Midwest Orthopaedics at Rush, Rush University Medical Center, 1611 West Harrison Street, Suite 300, Chicago,IL 60612,U.S.A.E-mail:nverma@rushortho.com

(C) 2020 THE AUTHORS. Published by Elsevier Inc. on behalf of the Arthroscopy Association of North America. This is an open access article under the CC BY-NC-ND license (http://creativecommons.org/licenses/by-nc-nd/4.0/).

2666-061X/20560

https://doi.org/10.1016/j.asmr.2020.10.006 together limiting the self-healing capacity of cartilage. ${ }^{1,2}$ Surgical intervention represents a fairly common treatment option, with an estimated incidence of 90 cartilage surgeries per 10,000 patients. ${ }^{3,4}$ Surgical options include restorative techniques (i.e., osteochondral grafts, autologous chondrocyte implantation), allowing for cells or tissue to re-create a normal chondral surface, or reparative measures (i.e., microfracture), in which the a natural healing response is induced to promote healing of smaller cartilaginous defects. Treatment algorithms have significantly evolved in the past decade, such that reparative techniques are generally considered in lower physical demand settings and restorative techniques are favored for larger chondral or osteochondral lesions (i.e., $>2-3 \mathrm{~cm}$ ) and in most highdemand patients depending on the timing of the surgical intervention. ${ }^{5}$ 


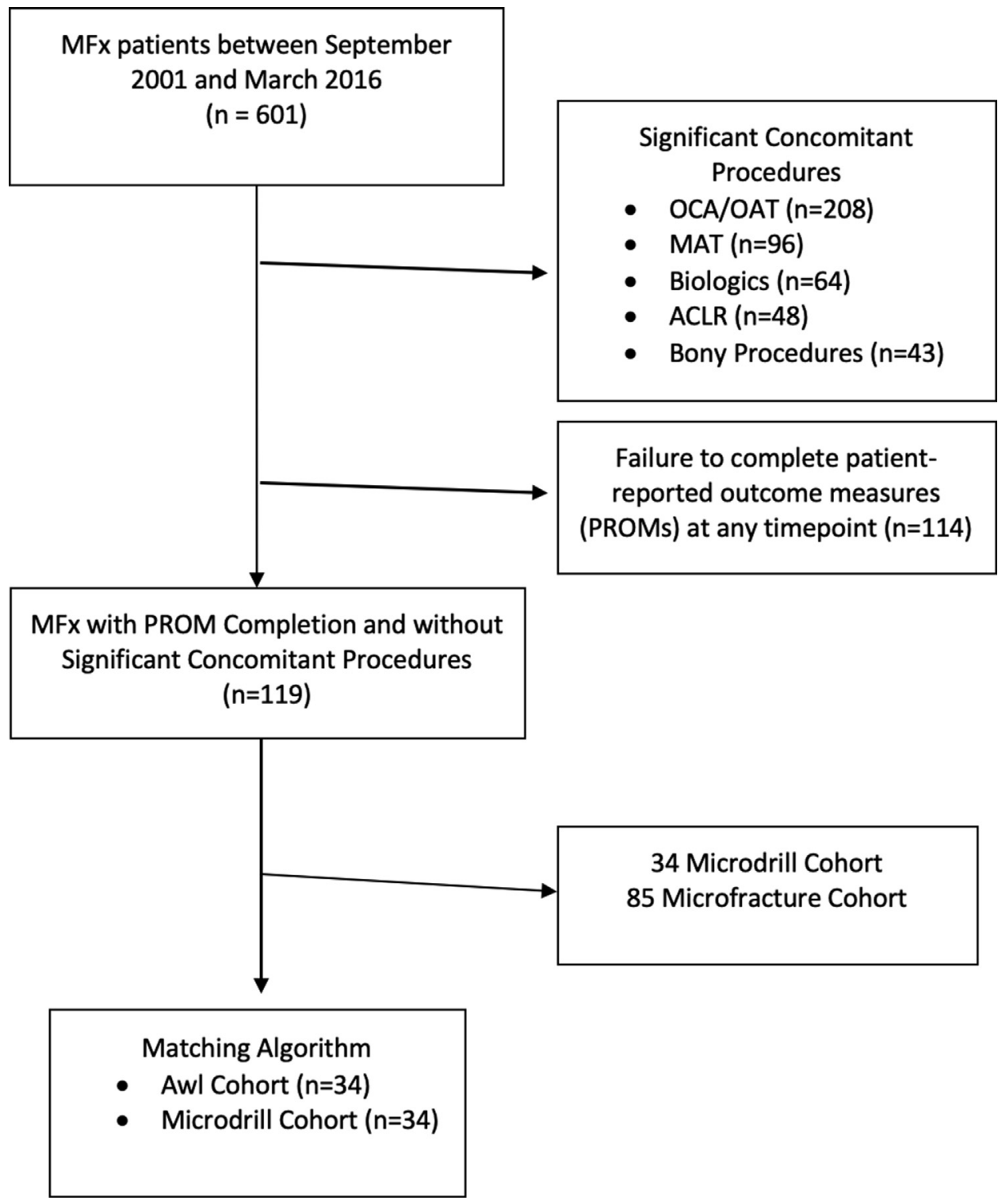

Fig 1. CONSORT diagram. Biologics include PRP, BMAC, and biocartilage. (ACLR, anterior cruciate ligament reconstruction; OAT, osteochondral autograft reconstruction; MAT, meniscal allograft transplantation; MFx, microfracture; OCA, osteochondral allograft reconstruction; PRP, platelet rich plasma; BMAC, bone marrow aspirate concentrate.)
The microfracture technique has been historically one of the most common procedures in the cartilage literature. $^{6,7}$ Marrow elements are released through the creation of small perforations in subchondral bone, resulting in the formation of a fibrocartilage layer that aids in defect repair. ${ }^{8,9}$ More recently, a microdrill (PowerPick; Arthrex, Naples, FL) has been introduced as a technical alternative to more traditional awl and hammer techniques. ${ }^{10,11}$ Histologic examinations of bone after treatment with a microdrill demonstrate decreased bony compaction and preservation of "bony channels" to marrow stroma relative to a traditional mallet and awl. ${ }^{12}$ However, the impact of technique variations on outcomes has yet to be determined, ${ }^{13-15}$ although based on histologic changes, improved outcomes may be expected.
The purpose of this study was to compare patientreported outcomes and revision rates between the standard microfracture awl versus the microdrilling technique. Our hypothesis was 2-fold: (1) no significant differences in outcome scores or the achievement of clinically significant outcomes scores and (2) revision rates at 1, 2, and 3 years would not differ between cohorts.

\section{Methods}

\section{Study Design and Cohort Establishment}

Our study cohort was constructed using a prospectively maintained institutional registry (Outcome Based Electronic Research Database; Universal Research Solutions, Columbia, MO). Patients undergoing 
microfracture surgery between September 2001 and March 2016 were screened using Concurrent Procedure Terminology codes for case identification. Inclusion criteria included receipt of microfracture cartilage surgery using either a standard microfracture awl versus microdrilling and completion of patient-reported outcome measures (PROMs) at preoperative, 6month, and 12-month time points. Exclusion criteria included concomitant meniscal pathology that required treatment (i.e., more than one-third of meniscal resection, meniscal repair or transplant), ligamentous (i.e., ligament reconstruction) and bony (i.e., osteotomy) procedures, and biological augmentation procedures (i.e., platelet-rich plasma, bone marrow aspirate concentrate injection). With respect to revision procedures, our definition of revision was a repeat surgery for the same lesion location as the primary procedure. A matching algorithm was run to match the demographic, preoperative, and intraoperative characteristics of the awl group to the PowerPick group (Fig 1).

\section{Surgical Technique}

Microfracture was performed according to previously described techniques. ${ }^{6}$ In short, the surgical technique involved debridement of the chondral defect, establishment of stable and vertical shoulders around the perimeter of the defect, removal of the calcified cartilage layer, and penetration of the subchondral bone with a microfracture awl or a 1.5-mm drill (PowerPick; Arthrex). The microfractured or microdrilled holes were evenly spaced 3 to $4 \mathrm{~mm}$ apart to preserve the structure and function of the subchondral bone plate. Marrow product egression (blood and fat droplets) was confirmed before the conclusion of each surgical procedure.

\section{Rehabilitation}

Postoperatively, the patients with tibiofemoral lesions were restricted to toe-touch weightbearing on the operative limb for the first 6 weeks, followed by gradual progression to full weightbearing after 6 weeks. Patients with patellofemoral lesions only were allowed to bear weight as tolerated immediately in a hinged knee brace locked in full extension. Patients were encouraged to use a continuous passive motion machine for 6 to 8 hours per day for 6 weeks. This protocol promoted adhesion and maturation of the fibrocartilage clot to the subchondral bone and has demonstrated clinical efficacy. ${ }^{16}$ Closed kinetic chain exercises were started at 2 weeks, and open kinetic chain exercises were started at 8 weeks. Impact exercises resumed at 4 to 6 months depending on patient symptoms, plyometric training at 5 months, and sport-specific drills (cutting, pivoting, twisting) at 6 months. The postoperative rehabilitation course was tailored to each individual patient based on
Table 1. Cohort Demographics and Patient-Reported Outcome Scores

\begin{tabular}{lccc}
\hline \multicolumn{1}{c}{ Characteristic } & Awl & Microdrill & $P$ Value \\
\hline Age, y & $34.1(30.7-37.5)$ & $28.5(23.8-33.2)$ & .09 \\
BMI & $27.5(25.6-29.4)$ & $25.9(24.3-27.5)$ & .29 \\
Sex & & & .63 \\
$\quad$ Male & $18(52.9)$ & $16(47.1)$ & \\
$\quad$ Female & $16(47.1)$ & $18(52.9)$ & .82 \\
Laterality & & & \\
$\quad$ Right & $19(55.9)$ & $17(50.0)$ & \\
Left & $15(44.1)$ & $17(50.0)$ & .57 \\
Previous surgeries & $1.74(1.1-2.4)$ & $2.08(1.5-2.7)$ & .11 \\
Lesion size & $2.65(2.0-3.3)$ & $1.93(1.6-2.3)$ & .49 \\
Lesion location & & & \\
$\quad$ LFC & $7(20.6)$ & $7(19.4)$ & \\
MFC & $8(23.5)$ & $13(36.1)$ & \\
Trochlea & $11(32.4)$ & $10(27.8)$ & \\
Patella & $4(11.8)$ & $2(5.6)$ & \\
MTC & $3(8.8)$ & $1(2.8)$ & \\
LTC & $3(8.8)$ & $2(5.6)$ & \\
CTC & $1(2.6)$ & $1(2.8)$ & \\
$\quad$ Bipolar lesions & $3(7.7)$ & $2(5.6)$ & \\
\hline
\end{tabular}

BMI, body mass index; CTC, central tibial condyle; LFC, lateral femoral condyle; LTC, lateral tibial condyle; MFC, medial femoral condyle; MTC, medial tibial condyle.

*Data reported as mean ( $95 \% \mathrm{CI}$ ) or as number (percentage). Fisher exact tests were performed for distribution of sex, laterality, and 3 location groups (femoral, patellar, or tibial), and Student $t$ tests were performed for age and BMI; all $\alpha=.05$.

symptoms, demand level, associated procedures, and the tempo of recovery.

\section{PROMs and Statistical Analysis}

Legacy PROMs of interest examined in this study include the International Knee Documentation Committee (IKDC) score; Knee Injury and Osteoarthritis Outcome Score (KOOS) subscales, including KOOS Pain, KOOS Symptoms (Sx), KOOS Activity of Daily Living (ADL), KOOS Sport and Recreational Activities, and KOOS Quality of Life (QOL); and Short Form 12 (SF12) Physical Component Score (PCS) and Mental Component Score (MCS). PROMs were administered at preoperative, 6-month, and 12-month time points. Statistical analysis examined differences in demographics using univariate statistical tests, including $\chi^{2}$ testing to examine the distribution of sex, laterality, and 3 location groups (femoral, patellar, or tibial). Student $t$ tests were performed to examine the distributions of age and body mass index (BMI) between the awl and microdrilling group. All statistical tests were performed at an $\alpha=.05$.

\section{Clinically Significant Outcomes}

Clinically significant outcome thresholds used in this study were adopted from previous literature for a variety of cartilaginous procedures. ${ }^{17}$ The minimally clinically important difference (MCID) was used in this study, 
Table 2. Preoperative PROM Scores Between Awl and Microdrilling Group

\begin{tabular}{lccc}
\hline Characteristic & Awl, Mean $(95 \%$ CI $)$ & Microdrill, Mean $(95 \%$ CI $)$ & $P$ Value \\
\hline IKDC & $42.9(37.4-48.4)$ & $44.4(39.0-49.8)$ & .76 \\
KOOS Pain & $59.3(53.5-65.1)$ & $59.9(55.0-64.8)$ & .89 \\
KOOS Sx & $62.0(57.6-66.4)$ & $62.3(57.1-67.5)$ & .94 \\
KOOS ADL & $73.3(67.4-79.2)$ & $37.7(29.8-45.6)$ & $.52 .75)$ \\
KOOS Sport & $31.5(23.9-39.1)$ & $27.5(20.5-34.5)$ & .16 \\
KOOS QOL & $27.4(21.4-33.5)$ & $37.2(33.5-40.9)$ & .49 \\
SF12 PCS & $40.9(38.5-43.3)$ & $53.3(49.2-57.4)$ & .09 \\
SF12 MCS & $47.5(42.5-52.5)$ & .09 \\
\hline
\end{tabular}

KOOS, Knee Injury and Osteoarthritis Outcome Score; IKDC, International Knee Documentation Committee; MCS, Mental Component Score; PCS, Physical Component Score; QOL, quality of life; SF12, Short Form 12; Sx, Symptom.

which represents the change in outcome score that results in the smallest, appreciable clinical improvement after surgery. ${ }^{18}$ When possible, thresholds for the MCID were derived from microfracture-specific cohorts (IKDC: 6.30). However, if threshold values for microfracture were unavailable, values from cartilage restoration procedures were used (i.e., KOOS Pain: 16.7, KOOS Sx: 3.6, KOOS ADL: 12.5, KOOS Sport: 12.8, KOOS QOL: 10.8, SF12 PCS: 6.5). ${ }^{17}$ Each of these values represented calculations using anchor-based methods with area under the curve values surpassing 0.7, except in the case of KOOS Sx, for which a distribution-based threshold was used. ${ }^{17}$ An anchor-based method employs a tiered questioning structure to determine levels of improvement, ${ }^{18}$ whereas a distribution-based method examines the distribution of patient-reported outcome scores to calculate an estimated MCID threshold value. ${ }^{19}$ Values from Ogura et al. ${ }^{17}$ were prioritized over other reports on MCID in microfracture due to no loss to follow-up in consecutive patient series. ${ }^{20} \mathrm{~A} \chi^{2}$ analysis was used to examine differences in percentage of patients reaching MCID at 6-month and 1-year time points between the awl and microdrilling groups.

\section{Results}

A total of 68 patients were included in our study population after the matching algorithm, without any significant differences observed between cohorts with respect to age, BMI, sex, laterality, number of previous surgeries, lesion size, or location $(P=.09-.82)$ (Table 1$)$. When examining mean preoperative scores between the awl and microdrilling groups, no significant differences in preoperative IKDC $(P=.76)$, KOOS subscales $(P=.33-.94)$, or SF12 PCS $(P=.09)$ or SF12 MCS $(P=.09)$ scores were observed (Table 2$)$.

At 6 months, the microdrilling group demonstrated significantly greater levels of improvement than the awl group on the IKDC $(64.6,95 \%$ CI $56.9-72.3$ vs 47.7 , $95 \%$ CI $41.9-52.0, P=.002)$, KOOS Pain $(76.1,95 \%$ CI 70.3-81.9 vs $67.9,95 \%$ CI 63.16-72.6, $P=.04)$, KOOS Sx $(75.6,95 \%$ CI $69.0-82.2$ vs $65.8,95 \%$ CI $60.2-71.4$, $P=.04)$, KOOS Sport $(55.2,95 \%$ CI $43.5-66.9$ vs 35, $95 \%$ CI $26.6-43.4, P=.01)$, KOOS QOL $(53.5,95 \%$ CI
42.4-64.6 vs $33.1,95 \%$ CI $25.4-40.8, P=.01)$, and the SF12 PCS $(46.2,95 \%$ CI $43.1-49.3$ vs $37.4,95 \%$ CI 34.8-40.0, $P=.002$ ). No significant differences between groups were observed at 6 months with respect to KOOS ADL $(86.0,95 \%$ CI 80.8-91.2 vs 79.2 , 95\% CI 72.9-85.5, $P=.10)$ or SF12 MCS $(54.0,95 \%$ CI 50.8 57.2 vs $56.1,95 \%$ CI 53.2-59.0, $P=.49$ ). At 1 year, no significant differences between the microdrill and awl groups were maintained with respect to IKDC (62.3, $95 \%$ CI $57.0-67.6$ vs $56.8,95 \%$ CI $48.7-64.9, P=.44)$, KOOS Pain $(74.22,95 \%$ CI 68.7-78.7 vs $75.7,95 \%$ CI 68.5-82.9, $P=.79)$, KOOS Sx $(72.6,95 \%$ CI 66.4-78.8 vs $72.6,95 \%$ CI 67.1-78.1, $P=.98)$, KOOS ADL (86.1, $95 \%$ CI $82.9-89.3$ vs $83.6,95 \%$ CI $77.0-90.2, P=.60)$, KOOS Sport (51.9, 95\% CI 44.1-59.7 vs 51.6, $95 \%$ CI 40.7-62.5, $P=.49)$, KOOS QOL $(54.2,95 \%$ CI $46.3-$ 62.1 vs $48.4,95 \%$ CI $38.2-58.6, P=.25)$, or SF12 MCS (55.9, $95 \%$ CI $53.4-58.4$ vs $54.9,95 \%$ CI $51.2-58.6$, $P=.15)$. Significant differences were maintained out until 1-year follow-up between groups for the SF12 PCS $(50.3,95 \%$ CI $48.6-52.0$ vs $38.4,95 \%$ CI 36.7-40.1, $P<.001$ ) (Fig 2).

\section{Rates of Reaching the MCID}

The rate of reaching the MCID for the awl group ranged from $6.7 \%$ to $50 \%$ at 6 months and $40 \%$ to $67.9 \%$ at 1 year by PROM. The range of percent reaching the MCID for the microdrilling group at 6 months was $11.8 \%$ to $72.0 \%$, with particularly high rates demonstrated on the IKDC instrument $(72 \%-91.7 \%)$. At 1 year, rates for the microdrilling group were $52.9 \%$ to $91.7 \%$. The microdrilling group demonstrated significant greater rates of reaching the MCID than the awl group at 6 months for the IKDC (72\% vs $33.3 \%, P=.002)$, KOOS Pain $(42.9 \%$ vs $11.1 \%, P=.02)$, and KOOS Sport $(55.6 \%$ vs $18.2 \%$, $P=.04)$. At 1 year, the microdrilling group also demonstrated significantly greater rates of reaching the MCID on the KOOS QOL $(83.3 \%$ vs $56.5 \%, P=.03)$ and SF 12 PCS $(90.9 \%$ vs $60.0 \%, P<.01)$. No significant differences were observed between the microdrilling and awl group at 6 months for KOOS QOL $(P=.17)$ or at 1 year for KOOS Sport $(P=.18)$, KOOS 


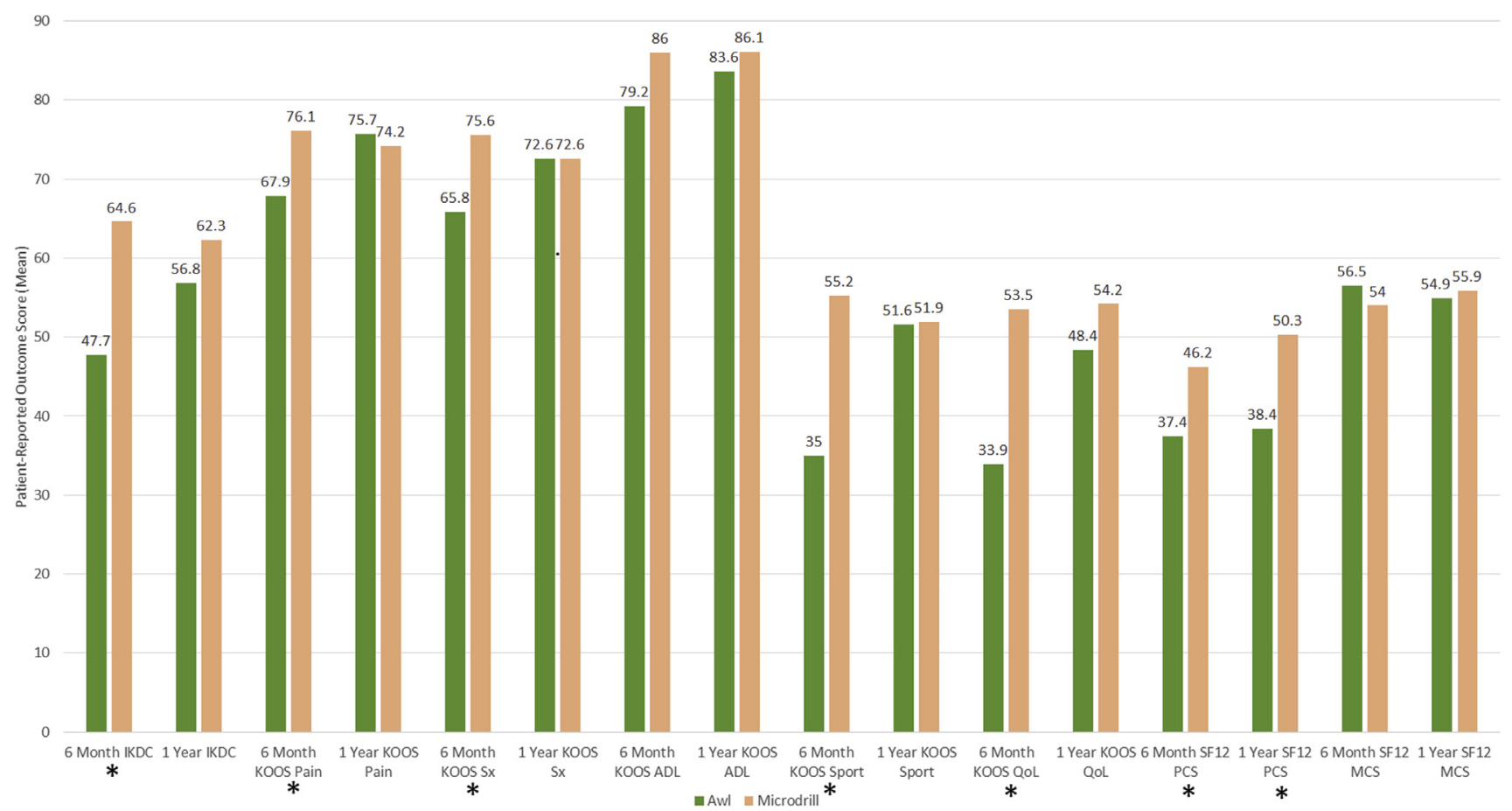

Fig 2. Patient-reported outcome scores across time points between groups. *Significant differences between groups with respect to score distributions at a specific time point on Student $t$ test at $\alpha=.05$. Significant improvements in postoperative PROM scores on one-way analysis of variance with $\alpha=.05$ were observed for all measures except SF12 MCS $(P=.12)$ and SF12 PCS $(P=.36)$. (IKDC, International Knee Documentation Committee; KOOS, Knee Injury and Osteoarthritis Outcome Score; MCS, Mental Component Score; PCS, Physical Component Score; QOL, quality of life; SF12, Short Form 12; Sx, Symptom.)

Pain $(P=.09)$, or IKDC $(P=.11)$. No significant differences between groups were found for KOOS Sx or KOOS ADL at 6 months or 1 year $(P=.11-.33)$ (Figs 3-4).

\section{Rates of Revision Surgery}

Within a 3-year period of follow-up, the awl group demonstrated a higher rate of revision surgery than the microdrilling group $(41.1 \%$ vs $17.6 \%, P=.02)$. Patients in the awl group were also more likely to require multiple subsequent procedures in the 3-year follow-up period than those in the microdrilling group (17.6\% vs $0 \%, P=.025)$. On subgroup analysis, there was a large difference in the percentage of reoperations within the first postoperative year between groups, although this failed to reach significance $(P=.16)$. There were no significant differences in the rate of subsequent surgery between groups in the first year $(P=.18)$, second year $(P=.82)$, or third postoperative year $(P=.63)$ (Fig 5).

With respect to the microdrilling cohort, the most common revision procedure was chondral debridement $(\mathrm{n}=4)$ follow by osteochondral allograft of either the
Fig 3. Achievement of minimally clinically important difference at 6 months. *Significant differences between groups with respect to score distributions at a specific time point on $\chi^{2}$ testing at $\alpha=.05$. Significant differences at 6 months were observed between groups for the IKDC, KOOS Pain, and KOOS Sport. (IKDC, International Knee Documentation Committee; KOOS, Knee Injury and Osteoarthritis Outcome Score; MCS, Mental Component Score; PCS, Physical Component Score; QOL, quality of life; SF12, Short Form 12; Sx, Symptom.)

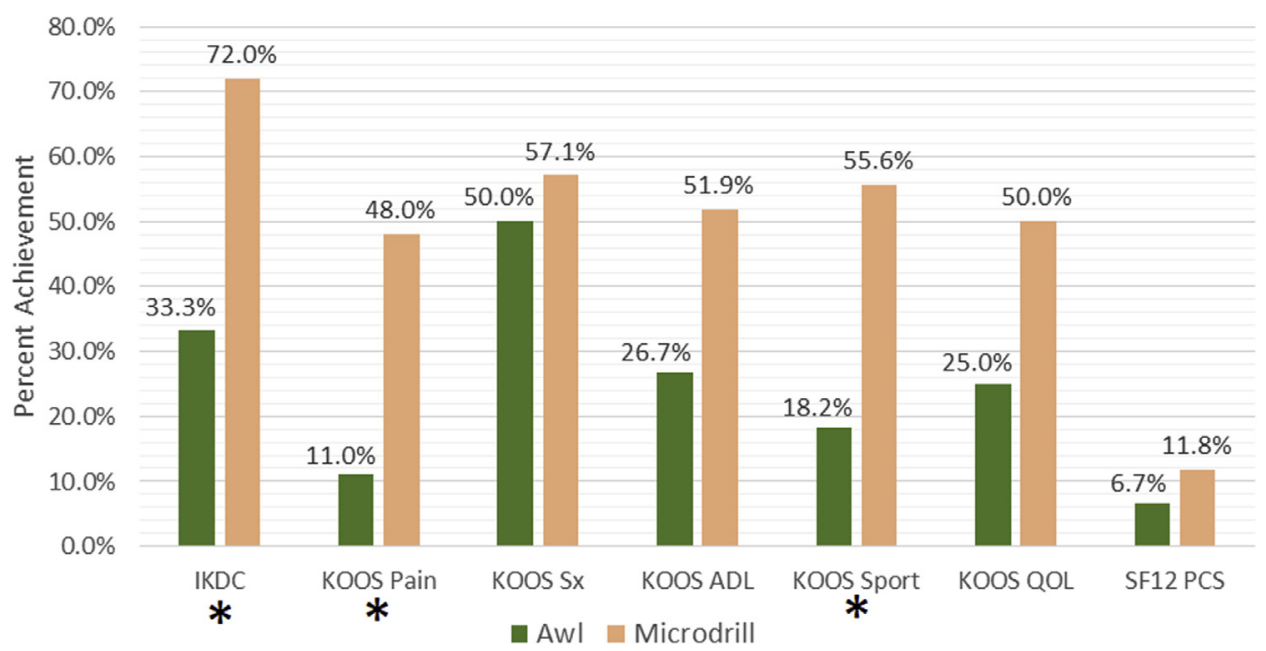




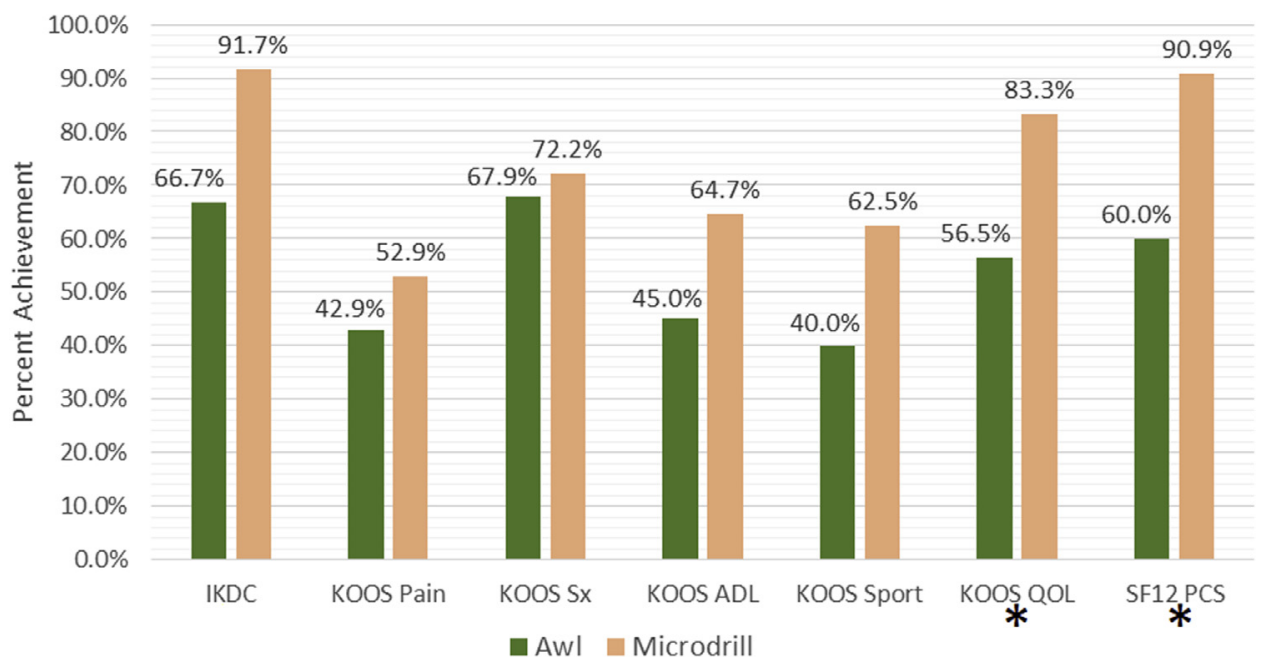

Fig 4. Achievement of minimally clinically important difference at 1 year. *Significant differences between groups with respect to score distributions at a specific time point on $\chi^{2}$ testing at $\alpha=$ .05. Significant differences at 1 year were observed between groups for KOOS QOL and SF12 PCS. (IKDC, International Knee Documentation Committee; KOOS, Knee Injury and Osteoarthritis Outcome Score; MCS, Mental Component Score; PCS, Physical Component Score; QOL, quality of life; SF12, Short Form 12; Sx, Symptom.) trochlea or femoral condyles $(n=3)$. Overall, $20.6 \%$ $(\mathrm{n}=6)$ of patients required subsequent surgery in the microdrilling group. Five patients in the awl cohort received a subsequent osteochondral allograft, with 6 requiring cartilage debridement and 2 requiring lateral release procedures. In addition, 3 patients received autologous chondrocyte implantation, 2 of which were performed with concomitant anteromedialization procedures. A single patient required patellofemoral arthroplasty, and another single patient in this cohort required an osteochondral autograft transplant. The overall revision rate at 3 years in this cohort is $47.1 \%$ $(\mathrm{n}=14)$.

\section{Discussion}

The main findings of this study demonstrated distinct short-term clinical advantages with respect to microdrilling relative to traditional mallet and awl impaction techniques used for microfracture surgery. Patients receiving microdrilling demonstrated significantly improved patient-reported outcome scores at 6 months on the IKDC, SF12 PCS, KOOS Pain, KOOS Sx, and KOOS QOL and at 1 year on the SF12 PCS instrument. This corresponded with increased rates of reaching the MCID at 6 months on the IKDC, KOOS Pain, and KOOS Sport PROMs. The traditional awl technique was associated with greater revision rates within 3 years of surgery and associated with the need for multiple revision surgeries. These findings suggest superior short-term outcomes for microdrilling with respect to traditional fracture techniques. Possible explanations include bony channels to marrow stroma, decreased bony compaction, and differences in osteonecrosis.

With respect to statistically significant differences in PROM scores between groups at specific time points, significant differences between the microdrilling and microfracture group were observed for the IKDC, KOOS Pain, KOOS Sx, KOOS Sport, KOOS QOL, and SF12 PCS. However, it is important to note that there were overlaps in the $95 \%$ confidence interval between
Fig 5. Awl versus microdrilling: rates of revision surgery.

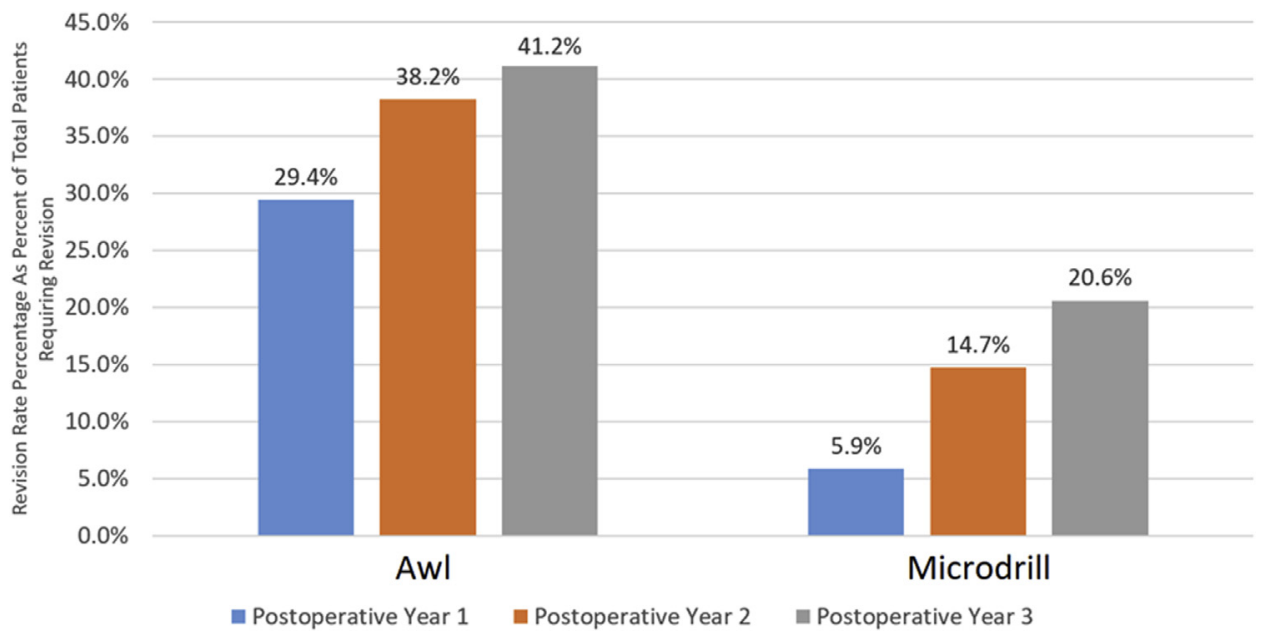


groups for the KOOS Sx and KOOS Pain, calling into question the clinical relevance of these findings. Together, the IKDC, KOOS Sport, KOOS QOL, and SF12 PCS represent the instruments for which there were significant differences between groups, whereas no statistically significant or clinically relevant differences were observed for KOOS Pain, KOOS Sx, KOOS $\mathrm{ADL}$, and SF12 MCS. These findings suggest that microdrilling may demonstrate superior functional outcomes for patients relative to microfracture, specifically with respect to sporting activities (i.e., stair climbing, running, jumping, twisting, kneeling) ${ }^{21,22}$ due to an overlapping of domains of assessment between IKDC, KOOS Sport, and SF12 PCS. No differences between were found in KOOS Sx or KOOS $\mathrm{ADL}$, suggesting both microdrilling and microfracture relieve symptoms to similar degrees and return patients to similar levels of daily activity. Both statistically and clinically significant outcomes after microfracture surgery using the traditional awl technique ${ }^{16}$ were demonstrated on the IKDC, SF12 PCS, and KOOS subscales (i.e., KOOS Pain, Sport, QOL) in this study. The results align well with previous studies that have demonstrated statistically significant improvements in similar instruments (i.e., IKDC, KOOS subscales). Randomized control trials examining longitudinal outcomes after microfracture have also demonstrated statistically significant improvements on various PROMs (i.e., IKDC, Lysholm, KOOS subscales) at postoperative time points. ${ }^{23-27}$ In addition, Weber et al. ${ }^{13}$ recently demonstrated both clinically and statistically significant improvements on Western Ontario and McMaster Universities Arthritis Index and KOOS instruments in microfracture patients after an average of 5.66 years of follow-up. However, Weber et al. ${ }^{13}$ only reported that mean differences in PROM scores surpassed MCID thresholds, whereas our study applied MCID thresholds to show superior clinically significant outcome achievement at 6 months on the IKDC, KOOS Pain, and KOOS Sport for the microdrilling cohort. This finding is particularly important given that outcomes after microdrilling have yet to be defined. Instead, most research on microdrilling in microfracture surgery has focused on defining histologic differences with respect to drill depth, thermal osteonecrosis, ${ }^{28,29}$ and bony architecture. ${ }^{12,30-33}$ The comparative analysis of outcome achievement across domains in this study suggests that function, pain, and sporting activities represent 3 clinical outcome domains for which microdrilling may provide an improved timeline of improvement relative to traditional awl microfracture. ${ }^{33}$

With respect to subsequent surgery, the microdrilling group in this study demonstrated significantly decreased rates of revision surgery at 3 years $(41.1 \%$ vs $17.6 \%)$ and decreased likelihood of requiring multiple surgeries $(17.6 \%$ vs $0 \%)$ relative to the awl cohort, respectively. Rates of subsequent surgery after microfracture using an awl and hammer technique have demonstrated increasing revision rates over time. ${ }^{13,34-36}$ Revision rates within the first postoperative year have been reported to range between $3 \%$ and $9 \%,{ }^{36}$ and 5 -year revision rates have been reported to increase to $22 \%$ to $42 \% .^{13,34,35}$ Weber et al. ${ }^{13}$ retrospectively reviewed outcomes in a cohort of patients receiving microfracture with an awl from the same institution. Longitudinal revision rates from that study are nearly identical to those in the awl cohort of the current study $(42.1 \%$ vs $41.2 \%))^{13}$ Although a handful of studies have compared revision rates between microfracture and autologous chondrocyte implantation, $^{23,24}$ the impact of microdrilling on revision rates after microfracture surgery has not been previously established.

Microfracture using the traditional awl technique is widely preferred over microdrilling due largely to low cost, technical ease, and concerns of thermal osteonecrosis. ${ }^{29,31,37,38}$ However, recent studies have demonstrated no increased propensity toward osteophyte necrosis when microdrilling is performed with irrigation. Although both the traditional awl and microdrilling techniques effectively perforate subchondral bone to allow migration of mesenchymal stem cells, $^{39}$ studies examining outcomes after the awl technique have demonstrated subsequent osteophyte formation, bone plate thickening, and subchondral cyst formation in up to one-third of patients. ${ }^{27,40,41}$ Other important limitations associated with the awl technique include bony overgrowth, ${ }^{41}$ bony compaction limiting access to marrow stroma, ${ }^{12}$ and subchondral sclerosis. ${ }^{41}$ These limitations align well with the results of our study because the awl technique demonstrated twice the rate of revision cartilage debridement as the microdrilling group $(17.6 \%$ vs $8.8 \%)$. In contrast, microdrilling has important advantages, including standardized drill depth (i.e., 4 to $6 \mathrm{~mm}$ depending on instrument), increased subchondral hematoma formation, and improved access to marrow stroma. ${ }^{12,31}$ Access to the marrow stroma is particularly important given evidence that microdrilling removes necrotic debris that has been demonstrated to block marrow stroma access in the case of awl microfracture. ${ }^{12}$ All in all, basic science studies exploring microdrilling in cartilage surgery suggest a mechanistic framework for the differences in clinical outcomes and revision rates observed in our study.

\section{Limitations}

The current study is not without limitations. First, MCID threshold values from cohorts of patients receiving cartilage reparative and/or restorative procedures were used given that microfracture-specific thresholds have not been established for most PROMs 
used in this study. Furthermore, this study did not report on survivorship to total knee arthroplasty or determine advancement of osteoarthritis by either clinical or radiographic parameters. Third, although we report revision rates between cohorts out to 3 years, we are only able to provide complete patient-reported outcome data out until 1 year. With respect to rehabilitation, the rehabilitation protocol in this study encouraged use of a continuous passive motion (CPM) machine for 6 to 8 hours a day for a period of 6 weeks. Variability in the use of CPM may contribute to the differences in clinical outcomes and revision rates observed between cohorts, particularly if the microdrilling cohort used the CPM significantly more or if the microfracture cohort used CPM significantly less. There may also be further variability with respect to rehabilitative status at the 6-month time point, depending on the patient's preexisting functional status. Our study design used a prospectively maintained database to retrospectively construct matched cohorts and examine outcomes. Historical control groups are problematic and could result in a type I error inflation. Consequently, a prospective design would be superior in terms of tracking outcomes and establishing rates of revision. Moreover, our matched cohort design yielded a $P$ value of .11 when attempting to control for lesion size between cohorts, raising concern for type II error. These lesion sizes represent an important limitation in our match cohort design because the mean size of the microfracture group differed from that of the microdrilling group by $27 \%$. This difference may be partly responsible for the group difference observed between cohorts in our analysis. Lesion location presents another limitation to our data set, as the microfracture group had 6 more patients with patellar, tibial, and trochlear defects, and these types of cartilage defects have been demonstrated to have inferior outcomes compared with femoral condyle lesions. ${ }^{42}$ It is also possible that in certain cases, there was new trauma in the same compartment or that the originally treated lesions propagated further. All in all, the concern of selection bias secondary to cohort differences in age, BMI, lesion size, lesion location, and rehabilitation remain.

In addition, a total of 3 surgeons performed microfracture surgeries across databases, creating potential variability with respect to rehabilitation and clinical decision-making protocols. However, when examining the distribution of surgeons as a proxy for clinical decision making and rehabilitation protocol variability, distributions were without significant differences between groups, suggesting limited provider bias $(P=.71)$. Last, although differences in bony morphology have been established between awl and microdrilling techniques, we were unable to examine differences in bony morphology at follow-up time points between groups. Important strengths to consider include revision surgery data in the subsequent 3 years of postoperative follow-up, analysis of both statistically and clinically significant outcomes, and the use of a matched cohort analysis to minimize preoperative variability with respect to demographic variables, lesion character (i.e., size, location, previous surgeries), and preoperative outcome scores that would possibly bias the study.

\section{Conclusions}

Microdrilling demonstrated superior outcomes relative to traditional microfracture awl techniques with respect to patient-reported outcomes at 6 months and revision rates within 3 years of follow-up. In addition, clinically meaningful differences were evident at 6 months in the microdrilling group.

\section{References}

1. Sophia Fox AJ, Bedi A, Rodeo SA. The basic science of articular cartilage: Structure, composition, and function. Sports Health 2009;1:461-468.

2. Gomoll AH, Minas T. The quality of healing: Articular cartilage. Wound Repair Regen 2014;22:30-38 (suppl 1).

3. McCormick F, Harris JD, Abrams GD, et al. Trends in the surgical treatment of articular cartilage lesions in the United States: An analysis of a large private-payer database over a period of 8 years. Arthroscopy 2014;30: 222-226.

4. Alford JW, Cole BJ. Cartilage restoration, part 1: Basic science, historical perspective, patient evaluation, and treatment options. Am J Sports Med 2005;33:295-306.

5. Farr J, Cole B, Dhawan A, Kercher J, Sherman S. Clinical cartilage restoration: Evolution and overview. Clin Orthop Relat Res 2011 1;469:2696-2705.

6. Steadman JR, Rodkey WG, Briggs KK. Microfracture: Its history and experience of the developing surgeon. Cartilage 2010;1:78-86.

7. Steadman JR, Rodkey WG, Rodrigo JJ. Microfracture: Surgical technique and rehabilitation to treat chondral defects. Clin Orthop Relat Res 2001;391:S362-S369.

8. Blevins FT, Steadman JR, Rodrigo JJ, Silliman J. Treatment of articular cartilage defects in athletes: An analysis of functional outcome and lesion appearance. Orthopedics 1998;21:761-768.

9. Gobbi A, Nunag P, Malinowski K. Treatment of full thickness chondral lesions of the knee with microfracture in a group of athletes. Knee Surg Sports Traumatol Arthrosc 2005;13:213-221.

10. Mirza MZ, Swenson RD, Lynch SA. Knee cartilage defect: Marrow stimulating techniques. Curr Rev Musculoskelet Med 2015;8:451-456.

11. Broyles JE, O'Brien MA, Stagg MP. Microdrilling surgery augmented with intra-articular bone marrow aspirate concentrate, platelet-rich plasma, and hyaluronic acid: A technique for cartilage repair in the knee. Arthrosc Tech 2017;6:e201-e206.

12. Chen H, Sun J, Hoemann CD, et al. Drilling and microfracture lead to different bone structure and necrosis 
during bone-marrow stimulation for cartilage repair. J Orthop Res 2009;27:1432-1438.

13. Weber AE, Locker PH, Mayer EN, et al. Clinical outcomes after microfracture of the knee: midterm follow-up. Orthop J Sports Med 2018;6:2325967117753572.

14. Berta A, Duska Z, Toth F, Hangody L. Clinical experiences with cartilage repair techniques: Outcomes, indications, contraindications and rehabilitation. Eklem Hastalik Cerrahisi 2015;26:84-96.

15. Negrin L, Kutscha-Lissberg F, Gartlehner G, Vecsei V. Clinical outcome after microfracture of the knee: A metaanalysis of before/after-data of controlled studies. Int Orthop 2012;36:43-50.

16. Steadman JR, Briggs KK, Rodrigo JJ, Kocher MS, Gill TJ, Rodkey WG. Outcomes of microfracture for traumatic chondral defects of the knee: Average 11-year follow-up. Arthroscopy 2003; 19:477-484.

17. Ogura T, Ackermann J, Mestriner AB, Merkely G, Gomoll AH. The minimal clinically important difference and substantial clinical benefit in the patient-reported outcome measures of patients undergoing osteochondral allograft transplantation in the knee. Cartilage 2019;28: P939-P948.

18. Cvetanovich GL, Gowd AK, Liu JN, et al. Establishing clinically significant outcome after arthroscopic rotator cuff repair. J Shoulder Elbow Surg 2019;28:939-948.

19. Rai SK, Yazdany J, Fortin PR, Avina-Zubieta JA. Approaches for estimating minimal clinically important differences in systemic lupus erythematosus. Arthritis Res Ther 2015;17:143.

20. Wang D, Chang B, Coxe FR, et al. Clinically meaningful improvement after treatment of cartilage defects of the knee with osteochondral grafts. Am J Sports Med 2019;47:71-81.

21. Roos EM, Lohmander LS. The Knee injury and Osteoarthritis Outcome Score (KOOS): From joint injury to osteoarthritis. Health Qual Life Outcomes 2003;1:64.

22. Collins NJ, Misra D, Felson DT, Crossley KM, Roos EM. Measures of knee function: International Knee Documentation Committee (IKDC) Subjective Knee Evaluation Form, Knee Injury and Osteoarthritis Outcome Score (KOOS), Knee Injury and Osteoarthritis Outcome Score Physical Function Short Form (KOOS-PS), Knee Outcome Survey Activities of Daily Living Scale (KOS-ADL), Lysholm Knee Scoring Scale, Oxford Knee Score (OKS), Western Ontario and McMaster Universities Osteoarthritis Index (WOMAC), Activity Rating Scale (ARS), and Tegner Activity Score (TAS). Arthritis Care Res (Hoboken) $2011 ; 63: S 208-S 228$ (suppl 11).

23. Knutsen G, Drogset JO, Engebretsen L, et al. A randomized trial comparing autologous chondrocyte implantation with microfracture: Findings at five years. J Bone Joint Surg Am 2007;89:2105-2112.

24. Gudas R, Kalesinskas RJ, Kimtys V, et al. A prospective randomized clinical study of mosaic osteochondral autologous transplantation versus microfracture for the treatment of osteochondral defects in the knee joint in young athletes. Arthroscopy 2005;21:1066-1075.

25. Basad E, Ishaque B, Bachmann G, Sturz H, Steinmeyer J. Matrix-induced autologous chondrocyte implantation versus microfracture in the treatment of cartilage defects of the knee: A 2-year randomised study. Knee Surg Sports Traumatol Arthrosc 2010;18:519-527.

26. Kon E, Gobbi A, Filardo G, Delcogliano M, Zaffagnini S, Marcacci M. Arthroscopic second-generation autologous chondrocyte implantation compared with microfracture for chondral lesions of the knee: Prospective nonrandomized study at 5 years. Am J Sports Med 2009;37:33-41.

27. Saris DB, Vanlauwe J, Victor J, et al. Treatment of symptomatic cartilage defects of the knee: Characterized chondrocyte implantation results in better clinical outcome at 36 months in a randomized trial compared to microfracture. Am J Sports Med 2009;37:10S-19S (suppl 1).

28. Lundskog J. Heat and bone tissue: An experimental investigation of the thermal properties of bone and threshold levels for thermal injury. Scand J Plast Reconstr Surg 1972;9:1-80.

29. Augustin G, Davila S, Mihoci K, Udiljak T, Vedrina DS, Antabak A. Thermal osteonecrosis and bone drilling parameters revisited. Arch Orthop Trauma Surg 2008;128: 71-77.

30. Hoemann CD, Sun J, McKee MD, et al. Chitosan-glycerol phosphate/blood implants elicit hyaline cartilage repair integrated with porous subchondral bone in microdrilled rabbit defects. Osteoarthritis Cartilage 2007;15:78-89.

31. Chen H, Chevrier A, Hoemann CD, Sun J, Ouyang W, Buschmann MD. Characterization of subchondral bone repair for marrow-stimulated chondral defects and its relationship to articular cartilage resurfacing. Am J Sports Med 2011;39:1731-1740.

32. Marchand C, Chen G, Tran-Khanh N, et al. Microdrilled cartilage defects treated with thrombin-solidified chitosan/blood implant regenerate a more hyaline, stable, and structurally integrated osteochondral unit compared to drilled controls. Tissue Eng Part A 2012;18:508-519.

33. Gao L, Goebel LKH, Orth P, Cucchiarini M, Madry H. Subchondral drilling for articular cartilage repair: A systematic review of translational research. Dis Model Mech 2018;11.

34. Frank RM, Cotter EJ, Nassar I, Cole B. Failure of bone marrow stimulation techniques. Sports Med Arthrosc Rev 2017;25:2-9.

35. Pestka JM, Luu NH, Sudkamp NP, et al. Revision surgery after cartilage repair: Data from the German Cartilage Registry (KnorpelRegister DGOU). Orthop J Sports Med 2018;6:2325967117752623.

36. Layton AAR, Graham J, Frasco MA, Cote E, Lynch NM. Long-term failure rates associated with knee microfracture surgery. J Int Soc Pharmoecon Outcomes Res 2015;18:A156.

37. Cole BJ, Pascual-Garrido C, Grumet RC. Surgical management of articular cartilage defects in the knee. J Bone Joint Surg Am 2009;91:1778-1790.

38. Matsunaga D, Akizuki S, Takizawa T, Yamazaki I, Kuraishi J. Repair of articular cartilage and clinical outcome after osteotomy with microfracture or abrasion arthroplasty for medial gonarthrosis. Knee 2007; 14: 465-471.

39. Shapiro F, Koide S, Glimcher MJ. Cell origin and differentiation in the repair of full-thickness defects of articular cartilage. J Bone Joint Surg Am 1993;75:532-553. 
40. Mithoefer K, Williams RJ III, Warren RF, et al. The microfracture technique for the treatment of articular cartilage lesions in the knee: A prospective cohort study. J Bone Joint Surg Am 2005;87:191 1-1920.

41. Brown WE, Potter HG, Marx RG, Wickiewicz TL, Warren RF. Magnetic resonance imaging appearance of cartilage repair in the knee. Clin Orthop Relat Res 2004: 214-223.

42. Kreuz PC, Steinwachs MR, Erggelet C, et al. Results after microfracture of full-thickness chondral defects in different compartments in the knee. Osteoarthritis Cartilage 2006;14:1119-1125. 